\title{
Fibrosis quística del páncreas
}

\author{
Estudio Clínico, de Laboratorio y Exploración Funcional Respiratoria. \\ Seguimiento a largo plazo \\ Dres.: Guillermo Vivanco (;.*, Aurelio Cuellar C.* Osvaldo Antelo A.* y Eliana Ceruti D.**
}

Si bien en Chile no se conoce la verdadera incidencia de la Fibrosis Quística (F.Q.), se sabe que en los países anglo-sajones tiene una alta incidencia de aproximadamente $1: 1000$ a $1: 2000$ recién nacidos vivos ${ }^{23}$. Por ser la enfermedad genética que más causa letalidad en esos países ha sido motivo de amplio estudio e investigaciones, sin embargo, el defecto básico permanece aún desconocido ${ }^{13-4-5 \hbar}$. El avance logrado en la expectativa de vida de estos pacientes hace razonable pensar que, su diagnóstico precoz y la aplicación subsecuente de medidas terapéticas eficaces, sean la causa de ello $^{35 i}$. Por todos estos motivos hemos creído interesante mostrar nuestra casuística, la más grande publicada hasta el momento en el país $^{3-9}$

\section{MATERIAL Y METODO}

Se analizan las principales características clínicas, de laboratorio y la evolución a largo plazo de 19 pacientes con F.Q., 10 de sexo masculino y 9 de femeuno, controlados todos en la Unidad de Enfermedades Respiratorias dei Hospital Roberto del Río.

El diagnóstico se estableció por el hallazgo de electrolitos en el sudor, sobre $60-70 \mathrm{mEq}$ de $\mathrm{Na}$ y Cl/litro, utilizando la técnica de iontoforesis por pilocarpina ${ }^{8} \cdot 10$. En 17 enfermos sc efectuaron un mínimo de dos exámenes seria-

\footnotetext{
* Residentes Depto. Pediatría Hospital Roberto del Rio.

** Profesor de Pediatría. Supervisor Unidad Enfermedades Respiratorias. Jefe Laboratorio Cardiorespiratorio Depto. Pediatría. Escuela de Medicina. Hospital Roberto del Río.
}

dos; en uno se efectuó sólo un examen porque el enfermo falleció en forma brusca; y en otro, el diagnóstico se hizo en la autopsia habiendo fallecido de un ileo meconial.

En 7 pacientes se efectuaron además sondeos duodenales ${ }^{11}$ y estudios de sobrecarga de caroteno ${ }^{12}$.

En todos se practicaron exámenes radiológicos de tórax seriados y estudios bacteriológicos de la secreción bronquial, tanto en los períodos controles como durante las complicaciones.

En 6 enfermos mayores de 6 años, se realizaron estudios funcionales respiratorios también seriados; la metodología de estos exámenes ya ha sido descrita ${ }^{13}$.

En este estudio se ha podido seguir y analizar la evolución de todos los pacientes desde su diagnóstico hasta el fallecimiento (10 enfermos) o su estado actual ( 9 enfermos).

\section{RESULTADOS}

Las características principales de los pacientes estudiados se muestran en la Tabla 1. En clla se destacan, la edad de comienzo de los síntomas, la edad del diagnóstico, el tiempo de la evolución pre y post diagnóstico y el estado actual de los enfermos (vivos o fallecidos). Se demuestra que en 7 pacientes los síntomas se presentaron ya en el período del Recién Vacido, en 7 durante el primer año de vida y en 5 en la etapa preescolar y escolar. En 9 enfermos el diagnóstico se efectuó tardíamente, después de un año de iniciada la enfermedad, a pesar de que habían consultado en numerosas oportunidades y que incluso habian estado hospitalizados en diversos servicios del país. En el momento de este 


\begin{tabular}{|c|c|c|c|c|c|}
\hline Pactentes & $\begin{array}{c}\text { Edad } \\
\text { iniciacion }\end{array}$ & $\begin{array}{c}\text { Tiempo ezolución } \\
\text { pre diagnóstico }\end{array}$ & $\begin{array}{c}\text { Edad } \\
\text { diagnóstico }\end{array}$ & $\begin{array}{l}\text { Tiempo evolución } \\
\text { post diagnóstico }\end{array}$ & $\begin{array}{l}\text { Estado } \\
\text { actual }\end{array}$ \\
\hline * 1 & R.N. & $2 \pi$ & $2 \mathrm{~m}$ & - & $F(2 m)$ \\
\hline$* * 2$ & R.N & $2 m$ & $3 \mathrm{~m}$ & $2 \mathrm{~m}$ & $V(5 \mathrm{~m})$ \\
\hline 3 & R.N. & - & $7 \mathrm{ds}$ & 2 a $8 \mathrm{~m}$ & $V\left(2 a^{8} m\right)$ \\
\hline 4 & R.N. & $105 \mathrm{~m}$ & 1 a $6 \mathrm{~m}$ & 6 a $1 \mathrm{~m}$ & $\mathrm{~F}(7 \mathrm{a} 7 \mathrm{~m})$ \\
\hline 5 & R.N. & $4 a$ & $4 \mathrm{a}$ & $9 \mathrm{a}$ & $F(13 a)$ \\
\hline 6 & R.N. & $8 m$ & $9 \mathrm{~m}$ & 5 a $8 \mathrm{~m}$ & $\mathrm{~F}(6$ a $5 \mathrm{~m})$ \\
\hline 7 & R.N. & $2 \mathrm{~m}$ & $3 \mathrm{~m}$ & - & $F(3 m)$ \\
\hline * 8 & $2 \mathrm{~m}$ & $7 \mathrm{~m}$ & $9 \mathrm{~m}$ & -. & $\mathrm{F}(9 \mathrm{~m})$ \\
\hline 9 & $3 \mathrm{~m}$ & $6 \mathrm{~m}$ & $9 \mathrm{~m}$ & & $F(9 \mathrm{~m})$ \\
\hline 10 & $3 \mathrm{~m}$ & $1 \mathrm{a} 5 \mathrm{~m}$ & 1 a $8 \mathrm{~m}$ & -- & $V(1$ a $8 \mathrm{~m})$ \\
\hline 11 & $3 \mathrm{~m}$ & $2 \mathrm{ag} \mathrm{m}$ & $3 a$ & 2 a $8 \mathrm{~m}$ & $V(5$ a $8 \mathrm{~m})$ \\
\hline$* * 12$ & $3 \mathrm{~m}$ & $3 \mathrm{ag}$ & $4 \mathrm{a}$ & - & $F(4 a)$ \\
\hline 13 & $6 \mathrm{~m}$ & $6 \mathrm{~m}$ & $1 \mathrm{a}$ & - & $F(1 a)$ \\
\hline 14 & $8 \mathrm{~m}$ & $3 a 4 m$ & $4 a$ & $1 \mathrm{a}$ & $\mathbf{F}(5 \mathrm{a})$ \\
\hline$* * * 15$ & 3 a $6 \mathrm{~m}$ & $3 a 6 m$ & $7 a$ & $11 \mathrm{a}$ & $V(18 a)$ \\
\hline$* * * 16$ & 7 a $4 \mathrm{~m}$ & - & $7 \mathrm{a} 4 \mathrm{~m}$ & $10 \mathrm{a}$ & $V(17 a)$ \\
\hline 17 & $8 \mathrm{a}$ & $6 a$ & $14 \mathrm{a}$ & $6 a$ & $V(20 a)$ \\
\hline$* * * 18$ & $9 \mathrm{a}$ & $3 a$ & $12 \mathrm{a}$ & $12 \mathrm{a}$ & $V(24 a)$ \\
\hline$* * * 19$ & $13 \mathrm{a}$ & - & $13 \mathrm{a}$ & $9 \mathrm{a}$ & $V(22 a)$ \\
\hline
\end{tabular}

* Pares de hermanos.

** Pares de hermanos.

*** Pares de hermanos.

estudio, 10 pacientes han fallecido y los restantes 9 tienen un período de evolución posterior al diagnóstico, que varía entre 2 meses y 12 años. Es importante hacer notar que 5 enfermos tienen una evolución mayor de 9 años. Cabe reparar que los pacientes que fallecieron fueron los que iniciaron más precozmente su sintomatología; en cambio aquellos en los que ésta apareció más tardíamente aún permanecen vivos. En esta casuística se apreció también en forma llamativa la presencia de 4 pares de hermanos.

En la mayoria de los enfermos, los síntomas y signos de la enfermedad pulmonar crónica fueron los predominantes. En un comienzo la enfermedad se caracterizó fundamentalmente por la presencia de tos y generalmente después de un a primera infección, el cuadro clínico se manifestó con signos que variaban en intensidad, pero que reflejaban siempre la presencia de una obstrucción bronquial generalizada. Además todos presentaban en mayor o menor grado signos y síntomas clínicos que revelaban la insuficiencia pancreática. Las deposiciones gene- ralmente eran más frecuentes en número, pastosas, grasosas y de mal olor, el abdomen estaba distentido y en los niños mayores de 4 años destacaba la presencia de dolor abdominal difuso o localizado en el epigastrio. El total de los niños de esta casuística mostraba grados diversos de desnutrición. Un enfermo inició su enfermedad con síntomas digestivos (síndrome de malabsorción) y posteriormente apareció el compromiso respiratorio. Dos enfermos de esta serie presentaron un ileo-meconio en el periodo de recién nacido, falleciendo uno de ellos en el transcurso de esta complicación.

En ninguno de los enfermos seguidos observamos evidencia clínica de cirrosis hepática.

En la Tabla 2 se muestran los valores de electrolitos en el sudor obtenidos en muestras seriadas en 18 de los niños estudiados. En todos, los valores fueron superiores a 60 a $70 \mathrm{mEq} /$ litro de Na y Cl; prácticamente todos tenían valores superiores a los $100 \mathrm{mEq}$ litro. Se observa además, que en muestras repetidas de un mismo enfermo los valores son similares. 
Tabla 2

TEST DEL SUDOR EN 18 PACIENTES CON F.Q."

\begin{tabular}{|c|c|c|c|c|c|c|}
\hline \multirow[b]{2}{*}{ Pacientes } & \multicolumn{2}{|c|}{$I$} & \multicolumn{2}{|c|}{ II } & \multicolumn{2}{|c|}{$I I I$} \\
\hline & $\mathrm{Na}$ & $C l$ & $N a$ & $\mathrm{Cl}$ & $\mathrm{Na}$ & $\mathrm{Cl}$ \\
\hline$* *$ & & & & & & \\
\hline 2 & 84,7 & 89,6 & 92,16 & 106,70 & & \\
\hline 3 & 132 & 125 & 128 & 127 & & \\
\hline 4 & 126,4 & 116,1 & 101 & 120 & & \\
\hline 5 & 132 & 125 & 127 & 125 & & \\
\hline 6 & 81,05 & 95,3 & 97,71 & 102,65 & 106,68 & 96,47 \\
\hline 7 & 120 & 110,5 & 118 & 118 & & \\
\hline 8 & 115,86 & 140 & 114,9 & 122,9 & & \\
\hline 9 & 132 & 123 & 128 & 127 & & \\
\hline 10 & 126,4 & 116,1 & 101 & 120 & $\cdot$ & \\
\hline 11 & 190 & 165 & 165 & 158 & & \\
\hline 12 & 104 & 126 & & & & \\
\hline 13 & 135,5 & 126,7 & 129,2 & 123,1 & & \\
\hline 14 & 75,76 & 133 & 126,6 & 144,9 & & \\
\hline 15 & 84 & 95 & 143 & 102 & 98 & 95 \\
\hline 16 & 116 & 112 & 122 & 133 & & \\
\hline 17 & 132 & 125 & 127 & 128 & & \\
\hline 18 & 111 & 102 & 117,8 & 109 & & \\
\hline 19 & 132 & 128 & 130 & 126 & & \\
\hline
\end{tabular}

* Los valores de $\mathrm{Na}$ y $\mathrm{Cl}$, se expresan en $\mathrm{mEq} / \mathrm{lt}$ de sudor.

* Paciente que fallece por ileo meconial a los $2 \mathrm{~m}$. Diagnóstico se hizo por autopsia.

En los 7 enfermos en los cuales se practicó sondeo duodenal se observó en todos aumento significativo de la viscosidad del líquido duodenal, disminución del volumen y ausencia de tripsina, lipasa, bicarbonato y amilasa. En ninguno se observó respuesta a la estimulación intraduodenal.

En forma parecida en los 7 pacientes en los cuales se efectuó un estudio de absorción de grasas medida por el test de sobrecarga de caroteno, se comprobó una deficiente absorción.

El examen radiológico de tórax fue relevante en todos los enfermos mostrando las diversas etapas del compromiso pulmonar. En una primera etapa, hiperdistensión y engrosamiento peribronquial, más adelante a medida que la enfermedad iba progresando se observó aumento del atrapamiento aéreo y áreas diseminadas de atelectasias y bronquiectasias y aumento sig nificativo de los engrosamientos peribronquiales. En las etapas terminales, especialmente en aquellos enfermos con evolución prolongada, se observaron evidèncias claras de fibrosis pulmonar. En ningún enfermo de esta serie se apreció neumotórax, ni abcesos pulmonares (Fig. 1).

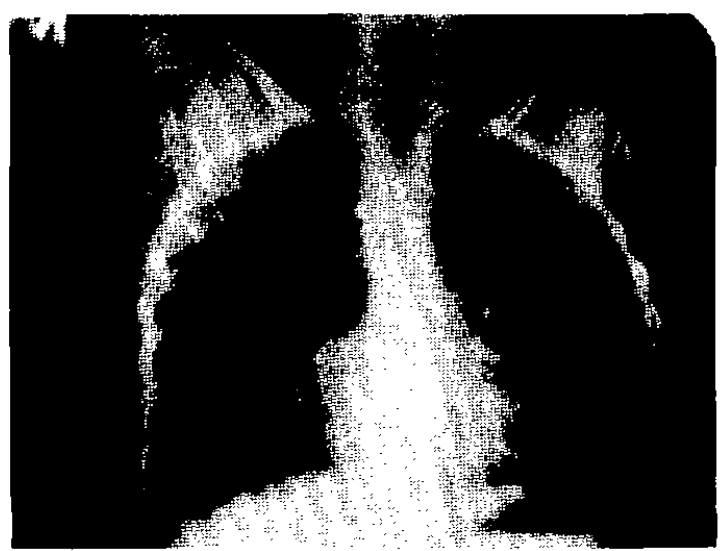

Fig. 1

Radiografía de tótax de una enferma de 5 aĩos con F.Q., se observan múltiples imágenes intersticiales, cordonales, areolares y peribronquiales parcialmente confluentes en la mayor parte del campo pulmonar derecho y en los $2 / 3$ inferiores izquierdos. Cisuritis horizontal derecha. Hiperdistensión pulmonar moderada.

Los estudios bacteriológicos seriados de secreción bronquial en muestras obtenidas espontán eamente o a través de aspirados traqueales o bronquiales, demostraron siempre la 
presencia de Staphylococcus aureus, asociado generalmente a Pseudomona Aeruginosa. En 3 enfermos se apreció una triasociación agregándose a los anteriores la presencia de Klebsiella Pneumoniae. Es importante consignar que la mejoría clínica no significaba siempre la erradicación del germen; más bien lo corriente de observar en los episodios intercurrentes era la mejoría clínica parcial y persistencia en el cultivo bronquial de uno de estos gérmenes especialmente del Staphylococcus y de éste más Pseudomona Aeruginosa en las etapas terminales.

En la figura 2 se analiza la evolución clínica que demuestra compromiso respiratorio predominante en 18 enfermos. Se observa que todos presentaron infecciones respiratorias intercurrentes que los llevaron progresivamente a la retención de secreciones, enfisema obstructivo y bronconeumonia y que finalmente produjeron un daño bronquial irreversible. Ocho pacientes han evolucionado hacia una enfermedad bronquial obstructiva crónica, leve a severa, y permanecen vivos. Un paciente está haciendo en este momento una bronconeumonia crónica progresiva; nueve han fallecido, cinco de Insuficiencia respiratoria total, y cuatro de insuficiencia respiratoria complicada con cor pulmonale.

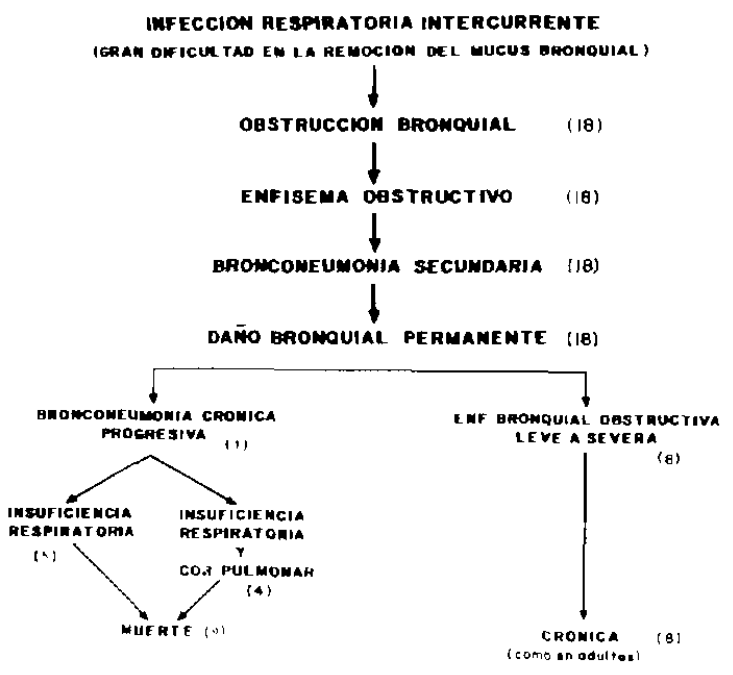

Fig. 2

Fibrosis Quística. Compromiso Respiratorio. Evolución de 78 pacientes

El estado actual de los 9 pacientes que permanecen vivos se muestra en la Tabla 3 , los

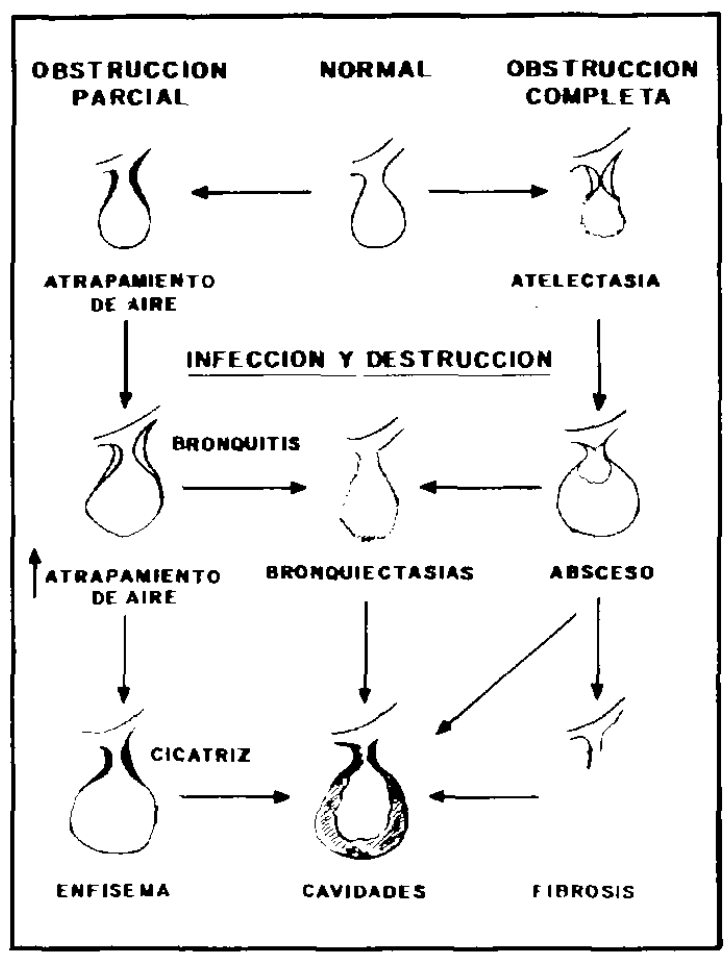

Fig. 3

Fibrosis Quística: Fisiopatología.

Tabla 3

ESTADO CLINICO ACTUAL EN 9 PACIENTES CON F.Q.*

\begin{tabular}{lrll}
\hline Grado & I & (Leve) & 3 \\
Grado & II & (Moderado) & 4 \\
Grado & III & (Grave) & 2 \\
\hline
\end{tabular}

"Clasificación adaptada de Jackson y Young.

pacientes se han agrupado de acuerdo al puntaje clínico descrito por Jackson y Young ${ }^{14}$ que los clasifica en base al estado general y hallazgos radiológicos en grado I: Buen estado general, bronquitis ocasionales o períodos cortos de tos. Radiografía de tórax normal o con grado mínimo de peribronquitis. Grado 11: Buen estado general con sintomas respiratorios permanentes, tos y desgarro de poca intensidad. Radiografía de tórax: alteraciones localizadas permanentes. Grado III: Compromiso severo del estado general, infección pulmonar persistente, con desgarro purulento, con signos pulmonares evidentes en la clínica y con Radiografía de tórax que revela alteraciones generalizadas. 
En nuestra casuística 3 pacientes fueron clasificados como leves (grado I), 4 moderados (grado II) y 2 graves (grado III).

Las complicaciones clínicas más relevantes se presentan en la Tabla 4. Entre las respiratorias llama la atención el alto número de atelectasias (10 enfermos) y bronquiectasias ${ }^{8}$. Entre las digestivas destacan la tinción del esmalte dental ${ }^{12}$ y los dolores abdominales ${ }^{6}$.

Tabla 4

19 PACIENTES CON F.Q.: COMPLICACIONES CLINICAS

\begin{tabular}{lr}
\hline Tipo de complicaciones & $N^{\circ}$ de pacientes \\
\hline Respiratorias & 10 \\
& 8 \\
Atelectasias & 4 \\
Bronquiectasias & 3 \\
Fibrosis pulmonar & 3 \\
Hemoptisis & 4 \\
Abcesos pulmonares & 2 \\
Cor pulmonar & \\
Deformaciones del torax & 12 \\
Digestivas & 6 \\
Tinción esmalte dental & 2 \\
Dolores abdominales & 1 \\
Heo meconial & \\
Pancreatitis recurrente & \\
\hline
\end{tabular}

Los diagnósticos diferenciales que se plantearon al inicio de la enfermedad se señalan en la Tabla 5. Se observa que en la mayoría de los enfermos se diagnosticaron Síndrome Bronquial Obstructivo y Bronconeumonías a repetición (7 casos), asociados a Síndromes de Malaborción y Enfermedad Celiaca en otros 4 casos.

Tabla 5

19 CASOS DE F.Q. DIAGNOSTICOS DIFERENCIALES

\begin{tabular}{lr}
\hline Diagnósticos & Pacientes \\
\hline Sínd. Bronquial Obstruc. BRN a repetición & 7 \\
Sínd. de Malabsorción. Enf. Celíaca & 4 \\
Ileo meconial & 2 \\
BRN de evolución prolongada TBC. & 1 \\
BRN de evolución prolongada. Bronquiectasias. & 1 \\
Bronquitis crónica obstructiva & 1
\end{tabular}

*En 3 pacientes el diagnóstico inicial fue F.Q. en base a antecedentes familiares.

En dos pacientes la enfermedad se inició en forma de ileo-meconio. Dos se catalogaron inicialmente como bronconeumonía crónica de evolución prolongada y otro como bronquitis crónica progresiva. En 3 enfermos el diagnóstico de F.Q. se planteó de inmediato ya que existían claros antecedentes familiares (hermanos afectados).

Los resultados del examen funcional respiratorio efectuado en los niños mayores de 6 años se muestran en la Tabla 6. Los valores promedios se expresan en porcentajes del valor

Tabla 6

EXPLORACION FUNCIONAL RESPIRATORIA EN 6 PACIENTES CON F.Q.

\begin{tabular}{lcrrrrrrrrrrrr} 
Enfermo & $C V$ & $C F R$ & $V R$ & $V R / C P T$ & $\% N_{2} A L V$ & $V E F_{1}$ & $C V F$ & $F E M$ & $F E M M$ & $E M F / V C$ & $P H$ & $P O_{2}$ & $P C O_{2}$ \\
\hline 15 & 68 & 180 & 220 & 64 & 4,8 & 59 & 62 & 55 & 58 & 52 & 7.385 & 53 & 45 \\
16 & 93 & 110 & 125 & 34 & 2,2 & 72 & 75 & 73 & 72 & 28 & 7.41 & 68 & 36 \\
17 & 37 & 97 & 164 & 57 & 6,7 & 46 & 48 & 33 & 22 & 48 & 7.32 & 46 & 52 \\
4 & 58 & 130 & 220 & 53 & 4,8 & 52 & 58 & - & - & 36 & & 41 & 54 \\
18 & 66 & 132 & 169 & 56 & 5,6 & 65 & 68 & 60 & 66 & 42 & 7.436 & 53 & 33 \\
5 & 57 & 96 & 199 & 49 & 3,6 & 38 & 56 & 35 & 31 & - & 7.36 & 48 & 46 \\
$\overline{\mathrm{X}}$ & 63 & 124 & 183 & - & 3.45 & 55 & 61 & 51 & 50 & & & - & - \\
\hline
\end{tabular}

CV : Capacidad Vital.

CPI : Capacidad pulmonar total.

$V_{\mathrm{VEF}} \quad$ : Volumen Espiratorio forzado primer segundo.

FEM : Flujo espiratorio máximo 200-1200.

EMF : Espacio muerto físiológico.

CFR : Capacidad funcional residual.

$\begin{array}{ll}\% \mathrm{~N}_{2} \mathrm{ALV} & \text { : Concentración } \mathrm{N}_{2} \text { Alveolar después de } 7 \text { de } \\ & 100 \% \mathrm{O}_{2} . \\ \text { GVF } & \text { Capacidad vital forzada. } \\ \text { FEMM } & : \text { Flujo espiratorio máximo medio } 25-75 \% . \\ \mathrm{VC} & : \text { Volumen corriente. } \\ \mathrm{VR} & : \text { Volumen residual. }\end{array}$

Los valores promedios de las capacidades, volúmenes y flujos respiratorios se expresan como porcentajes del valor teórico normal para cada niño. 
teórico normal. Se comprueba disminución progresiva de la capacidad vital, aumento de la capacidad funcional residual, del volumen residual y de la relación volumen residual/capacidad pulmonar total; por otra parte, se observa disminución significativa del volumen espiratorio forzado, primer segundo y de los flujos espiratorios máximos y máximos medios. Se destaca además que la relación espacio muerto fisiológico/volumen corriente está aumentada. Todos presentan a través del tiempo grados progresivos de hipoxemia e hipercapnea en las etapas avanzadas de la enfermedad.

El tratamiento efectuado en todos los enfermos con F.Q. se esquematiza en la Tabla $7^{15}$.

\section{Tabla 7}

TRATAMIENTO DE LA FIBROSIS QUISTICA

1. Dietético:

- Régimen rico en proteínas y calorías. Pobre en grasas.

- Aporte de triglicéridos de cadena mediana.

- Suplemento enzimático. Viokase (R), Ciotazym (R)

- Vitaminas: E, 1 a $5 \mathrm{U} \times \mathbf{K g} /$ día.

- Vitaminas: K, 5 a 10 mgs. 1 vez a la semana.

- Vitaminas: A y D, dosis habituales.

-Suplemento de sal: 2-4 gramos diarios.

- Bicarbonato de sodio: 1 a $4 \mathrm{~g}$. diarios.

2. Kinéstos:

- Drenaje postural

- Percusión y vibraciones torácicas.

- Ejercicios respiratorios.

- Actividad física.

3. Teropia humidificante:

- Tiendas.

- Nebulizaciones:

S. Fisiológico.

Antibióticos

Mucolíticos.

4. Antibióticos:

- Tetraciclina y Dicloxacilina uso ambulatorio y permanente.

- Otros antibióticos, pacientes hospitalizados, según estudio bacteriológico. Carbenicilina, Gentamicina, Tobramicina, Amikacina, Sisomicina, Cloxacilina

5. Oxigenoterapra controlada.

- En etapas finales.

6. Bronco aspiración:

- Casos seleccionados.
A todos los pacientes se les indicó regimen hiperproteico, hipercalórico e hipograso, dándoles un aporte extra de aceite a base de triglicéridos de cadena mediana y enzimas pancreáticas en cada comida. Además se indicaron vitaminas liposolubles (A, D, E, K), suplemento de sal y aporte extra de bicarbonato de sodio. Todos se sometieron a una terapia kinésica intensiva y permanente que incluía drenaje postural, percusión, vibraciones torácicas y ejercicios respiratorios. Durante las reagudizaciones se consideró fundamental el uso de humificadores. Los antibióticos se usaron de acuerdo a las necesidades de los pacientes y según antibiograma. La insuficiencia cardíaca que presentaron algunos enfermos en etapas terminales se trató de acuerdo a normas habituales en estos casos. En algunos pacientes muy seleccionados se practicaron una o varias broncoaspiraciones ${ }^{16}{ }^{17} y$ en los pacientes graves se indicó de regla oxigenoterapia controlada.

\section{DISCUSION}

La F.Q. del Páncreas es una enfermedad relativamente nueva que fue descrita por primera vez en $1936^{\circ}$. Es una enfermedad hereditaria transmisible como enfermedad recesiva que compromete niños, adolescentes y adultos jóvenes y que se caracteriza por una disfusión generalizada de las glándulas exocrinas. Se presenta como una triada con enfermedad pulmonar crónica, deficiencia pancreática y concentración anormalmente alta de electrolitos en el sudor ${ }^{134.5 .6}$

Entre sus características vale la pena destacar en primer lugar que no es una enfermedad que afecte siempre y en forma exclusiva al páncreas, por lo tanto, puede estar presente en enfermos que no evidencien insuficiencia pancreática; en segundo lugar, que no se trata de una enfermedad rara; tal vez en nuestro medio su incidencia hasta ahora ha sido baja, pero tanto en Estados Unidos, como en Canadá y Europa tiene una incidencia de $1 \times 1.000$ a $1 \times 2.000 \mathrm{na}$ cidos vivos ${ }^{12-3}$. En tercer lugar, que siempre es una enfermedad severa con gran diversidad de síntomas y signos clínicos, que afecta gravemente el aparato respiratorio en casi todos los pacientes, y que aún más, la mayor parte de las muertes están ocasionadas por las compli- 
caciones respiratorias. En cuarto lugar, es importante recalcar que un número no despreciable de estos enfermos alcanza la edad adulta, lo que evidentemente hace plantear la posibilidad que la incidencia de la enfermedad vaya aumentando, afortunadamente en contra de este fenómeno está el hecho que se ha demostrado que aproximadamente el $97 \%$ de los enfermos de sexo masculino son estériles ${ }^{19-20}$; finalmente debe señalarse que el diagnóstico es relativamente fácil, indoloro y de bajo costo ${ }^{10}$

La baja incidencia con que vemos esta enfermedad en nuestro medio, no tiene una explicación clara ${ }^{9.21}$ por lo que cabría plantearse dos posibilidades: la primera, razones de tipo genético ya que es conocido el hecho que existe una marcada predisposición por parte de algunos pueblos, especialmente los anglosajones ${ }^{1-3}$ y en cambio, en otros como negros, orientales e indios americanos ${ }^{22}$, prácticamente no existe. La segunda, por diagnóstico deficiente; al respecto, una revisión de autopsias realizadas en hospitales pediátricos de Santiago entre 1971 y 1976 demuestra que de 19 casos de fibrosis quística encontrados en sólo 7 de ellos el diagnóstico se formuló clínicamente ${ }^{21}$. Además, el hecho de que la F.Q. se pueda presentar como patología habitual en nuestro medio, especialmente en lactantes y niños menores, ya sea en forma crónica como aguda (bronconeumonías, diarreas, desnutrición) dificulta aún más el diagnóstico diferencial; a esto se suma que la mayoría de los pacientes con F.Q. inician su sintomatología precozmente, por cuya causa se omite el diagnóstico y es justamente este grupo el que presenta mayor mortalidad ${ }^{23}$.

En esta casuística hemos encontrado la presencia de 4 pares de hermanos. La incidencia de hermanos no debería ser mayor de un $25 \%$ $\sin$ embargo, es frecuente encontrar familias con 2 o más hermanos afectados. Este hecho se ha interpretado como simple casualidad, pero no se ha podido descartar la posibilidad de que exista algún factor que aún desconocemos y haga variar la expresividad genética.

La etiología de la F.Q. es oscura y confusa a pesar de lo mucho que se ha investigado al respecto. Se sabe que es hereditaria y no puede reproducirse en animales. Se postula que el desorden básico consistiría en una alteración de la membrana basal de las glándulas, acompaña- da de una disfunción del metabolismo celular que afectaría especialmente la función de las enzimas lisozomales comprometidas en la sintesis de la mucoproteina. Se ha postulado además, que existiría un defecto en la bomba de sodio $^{6.24-25}$. Estos fenómenos serían responsables del defecto primario de la enfermedad, es decir, del aumento de la viscosidad de las secreciones y del aumento del contenido de sal en las glándulas exocrinas.

Los órganos más comprometidos son los pulmones y el páncreas, hecho que comprobamos en todos nuestros enfermos. La estructura pulmonar usual con que nacen estos enfermos ${ }^{26}$ se altera por sucesivas infecciones respiratorias las que finalmente llevan a lesiones irreversibles que perpetúan y agravan cada día más la enfermedad.

La obstrucción de la vía aérea, tal como se observó en nuestros pacientes, puede ser parcial o completo. Si es total, se producen atelectasias que pueden evolucionar hacia la formación de abcesos y éstos hacia bronquiectasias, cavidades y fibrosis. La obstrucción parcial favorece el atrapamiento aéreo y la infección con daño secundario de la pared bronquial, factores que también pueden derivar en formación de bronquiectasias y cavidades o bien lesionar la pared bronquial con cicatrización posterior de ésta y aparición subsecuente de áreas de enfisema las cuales también pueden llegar a la formación de cavidades.

La enfermedad evoluciona rápida o lentamente hacia el deterioro progresivo, marcando el compromiso pulmonar el destino de estos pacientes ${ }^{1-3-4-5-14-17-26-27.28}$. Se crea un verdadero círculo vicioso, en que sucesivas infecciones pulmonares van ocasionando daños estructurales irreversibles, los que a su vez, facilitan nuevas infecciones. Durante las reagudizaciones que duran semanas o meses se observan grados variables de insuficiencia respiratoria. En los enfermos con F.Q. la gravedad de los episodios no se relacion a ni con la duración total de la enfermedad, ni con la edad del paciente; sin embargo, si el número de episodios aumenta, las complicaciones fatales también lo hacen.

La evaluación respiratoria, debe realizarse en cuanto sea posible, puesto que ella nos revela precozmente el compromiso respiratorio, aún 
antes que aparezcan los signos radiológicos. Nuestros resultados muestran un Pattern de trastornos físiológicos constantes, que está de acuerdo con lo observado por otros autores ${ }^{29-30}$ 31-32. Existe una enfermedad bronquial obstructiva que va aumentando progresivamente. Inicialmente puede ser reversible; pero, en etapas avanzadas, es irreversible agregándose compromiso de la perfusión pulmonar.

Tal como observamos en este estudio la mayor parte de estos niños cursan con hipoxemias crónicas, la $\mathrm{PaCO}_{2}$ es normal o baja, siempre que existan áreas pulmonares bien ventiladas. Durante las exacerbaciones la $\mathrm{PaCO}_{2}$ se eleva y demora semanas o meses en bajar. En los enfermos fallecidos se aprecian los valores máximos de hipoxemia e hipercapnia, en todos ellos la acidosis respiratoria se combina con acidosis metabólica.

El estado de insuficiencia respiratoria en la F.Q. debe ser reconocido y documentado, para evaluar la eficacia del tratamiento y reconocer complicaciones precoces. En esta enfermedad, por desgracia, las medidas terapéuticas son menos útiles que en otras formas de Insuficiencia Respiratoria. A esto cabría agregar lo oneroso y difícil que es en nues tro medio poder cumplir con todas las indicaciones de tratamiento. Por todo lo anterior manejar esta enfermedad, en que el defecto básico es desconocido, resulta siempre frustrante.

La revisión de la literatura demuestra compromiso pancreático en el $85 \%$ de los enfermos de F.Q. ${ }^{1-3-4-6-11-12-17-18-14-27}$. La insuficiencia pancreática se manifiesta por aquilia y aumento de la viscosidad del líquido duodenal y disminución de todas las enzimas. Fxiste esteatorrea y pérdida excesiva de grasa, nitrógeno y vitaminas liposolubles. En contraste, las proteínas se toleran bien lo que hace posible alcanzar un balance de proteinas positivo. Si bien en nuestra casuística sólo pudimos objetivar la insuficiencia pancreática en 10 pacientes (sondeo duodenal y test de sobrecarga de caroteno) porque en los restantes no fue posible realizar este tipo de estudio, ya sea porque rechazaron la sonda duodenal o no dispusimos en ese momento de estos exámenes de laboratorio o bien porque fallecieron antes de su realización, todos los enfermos presentaban síntomas y signos físicos de insuficiencia pancreática, especialmente est eatorrea y dolores abdominales.

En nuestra experiencia 2 enfermos presentaron ileomeconio, uno de ellos falleciendo de inmediato; estos hechos coinciden con los observados en grandes grupos de pacientes con F.Q. en los cuales se ha demostrado una incidencia de ileomeconio de $10 \%^{1.33}$.

Se describe en esta enfermedad que en un $15 \%$ de los pacientes existe compromiso hepático, cirrosis, hipertensión portal, várices esofágicas, hiperesplenismo ${ }^{34}$. En este estudio no observamos ninguna de estas complicaciones, por lo menos, desde el punto de vista clínico.

Es importante señalar que en la F.Q. el $97 \%$ de los hombres son estériles por defectos en el desarrollo de los conductos de Wolf, vasos deferentes, epididímo y vesículas seminales ${ }^{1920}$. También se ha observado que un $80 \%$ de las mujeres son estériles por aumento de la viscosidad y deshidratación de la secreción mucosa y presencia de útero infantil ${ }^{35}$

El diagnóstico de certeza de la enfermedad se hace mediante el test de electrolitos del sudor 1-4-8-10-17-27-36 el cual está francamente alterado en el $98 \%$ de los pacientes. Para que tenga el valor asignado debe ser realizado por lo menos 2 o 3 veces consecutivas en el mismo paciente y debe ser determinado con una técnica muy minuciosa y estandarizada, en un laboratorio de mucha experiencia. Debe tenerse en cuenta que los valores de sodio y cloro son más elevadas en las 2 primeras décadas de vida, posteriormente descienden y ya después de los 17 años de edad se alcanzan los valores del adulto normal, que son cerca del doble de los del nino ${ }^{6}$.

Actualmente existen además, otros dos procedimientos de laboratorio en uso, el primero, la determinación de la conductividad y el segundo la aplicación directa de un electrodo cutáneo para determinar el nivel de cloruros ${ }^{6} 37$. Sin embargo ambos métodos sólo han resultado confiables en manos de expertos.

Con la sola excepción de la insuficiencia suprarrenal y la diabetes insipida renal, no existe ninguna otra enfermedad en la que se obtengan los valores observados en las F.Q.

Junto con la determinación de los electrolitos en el sudor se debe en lo posible determinar el grado de compromiso pancreático. Para estos 
fines se realizan sondeos duodenales, puede efectuarse determinación de tripsina en las deposiciones, test de absorción de grasas, test de absorción de la vitamina $\mathrm{A}$ y test de absorción de lipiodol.

En la F.Q. el diagnóstico oportuno es básico y no debe esperarse a estar frente a un enfermo extraordinariamente grave o crónico para pensar en esta enfermedad. Debe sospecharse en todos los lactantes o niños que tengan síntomas recurrentes o crónicos que comprometan el aparato respiratorio alto o bajo, en los recién nacidos con ileo meconio, en enfermos cuya sintomatología haga pensar en insuficiencia pancreática o malabsorción y en niños con fallas del crecimiento, gran apetito, abultamiento intestinal, prolapso rectal, deficiencia de Vitamina K, etc. Con respecto a la oportunidad del diagnóstico debe siempre tenerse presente que la severidad de los síntomas varían grandemente de un enfermo a otro; mientras más tardío es el comienzo de la sintomatología respiratoria mayor es la sobrevida del enfermo. En los últimos años se han desarrollado algunas técnicas para detectar la F.Q. precozmente en grupos de recién nacidos; la más simple y confiable es la que utiliza la determinación de los niveles de albúmina en el meconio, "BMC-Test meconium strip". Desgraciadamente este método no se ha utilizado en nuestro país aun cuando ha significado un avance importante en el diagnóstico precoz de la enfermedad en grandes grupos de pacientes ${ }^{38-39}$.

La etiología de la F.Q. continúa siendo desconocida a pesar de las numerosas investigaciones que se han realizado. Se han descrito 3 mecanismos básicos ${ }^{256}$. 1. alteración de la membrana basal de las glándulas exocrinas, fundamentalmente disfunción en la reabsorción del sodio; 2 alteración del metabolismo de las mucinas que secundariamente afectaría el páncreas, los pulmones, etc. Se postula que las mucinas y glicoproteínas de las secreciones mucosas tendrían una estructura anormal que consecuentemente alteraría las propiedades físicas del mucus que tapiza las membranas. Este hallazgo junto con la determinación de un menor contenido de agua de la mucina ha sido confirmado en todas las investigaciones y 3 . alteración del mecanismo mucociliar o motor ciliar de glándulas y conductos; en los pacientes con F.Q. existiría una falla hereditaria del clearance ciliar. Se ha demostrado que aun en presencia de mucus normal existe un factor en el suero de los pacientes con F.Q. tanto heterozigotos como homocigotos que altera el movimiento ciliar normal de la tráquea del conejo; se ha llamado factor de disquinesia ciliar. Se presume que en los pacientes con F.Q., este factor alcanza rápidamente la mucosa cuando ést a se inflama por una infección.

El futuro de esta trágica enfermedad se basa en la investigación de sus causas fundamentales $y$ en el desarrollo de métodos confiables que permitan una prevención primaria, es decir, que detecten los individuos heterozigotos para permitir al médico dar consejo genético oportuno a los padres, o que faciliten el diagnóstico prenatal "in utero" de la enfermedad, de tal manera, de tomar una decisión al respecto. Por el momento, todas estas alternativas están en pleno desarrollo, son altamente promisorias, pero aún no muestran resultados específicos $y$ concluyentes $^{40}$ y por lo tanto, sólo disponemos de la prevención secundaria que es aconsejable en países con una incidencia importante de F.Q. Al respecto, deben desarrollarse métodos altamente específicos y reproducibles que permitan detectar precozmente la enfermedad, ojalá en R.N. y lactantes, y de esta manera, permitir la realización de un tratamiento oportuno y a largo plazo.

\section{RESUMEN}

1. Se analizan las principales características clínicas, de laboratorio y la evolución a largo plazo de 19 pacientes con F.Q.; 10 varones, 9 mujeres.

2. En todos, el diagnóstico se confirmó por el hallazgo de electrolitos en el sudor elevados. En todos se efectuaron estudios Radiológicos de Tórax y estudios bacteriológicos de la secreción bronquial. Fn siete enfermos se efectuaron además sondeos duodenales y test de sobrecarga de caroteno. En seis enfermos mayores de 6 años se efectuaron estudios funcionales respiratorios seriados.

3. Se describe la evolución a largo plazo ( 2 meses a 12 años) y el estado actual de los enfermos ( 9 vivos y 10 fallecidos).

4. Se destaca que en todos los enfermos menos uno, que falleció de ileo meconia en el período de Recién Nacido, los síntomas y signos de la enfermedad julmonar crónica fueron los predominantes. 
5. Todos los enfermos en mayor o menor grado presentaron signos y sintomas de insuficiencia pancreatica. En ninguno de los enfermos seguidos hubo evidencias clínicas de compromiso hepático.

6. El examen funcional respiratorio revela enfermedad bronquial obstructiva progresiva

7. Se analizan las complicaciones clínicas más reievantes y los principales diagnósticos diferenciales planteados al inicio de la enfermedad.

8. Se describe el tratamiento efectuado en el total de los enfermos estudiados.

9. Se actualizan los conocimientos básicos acerca de la etiología de la enfermedad y se comentan los nuevos ca. minos para llegar a su prevención.

\section{REFERENCIAS}

Guide to diagnosis and management of Cystic Fibrosis, National Cystic Fibrosis Research Foundation, 1975.

2 Huang, N.; Macre, C.: Girone, J.; Sproul, A. Survival of patients with cystic fibrosis. Amer. 3. Dis. Child. 120:289, 1970.

3 Crozier, D.N. Cystic Fibrosis a not so fatal disease. Ped. Clin. N. Amer. 21:935, 1974.

1 Di Sant'Agnese, P.A. Cystic Fibrosis (Mucovisidosis). Amer. Fam. Physician 7:102, 1973

5 Warwick, W.J. Report to Canadian Cystic Fibrosis. Aug. 23, 1973.

6 Report of the Committee for a study for evaluation of testing for Cystic Fibrosis. J. Pediatr. 88:711, 1976.

-7 Martínez, F.; Moreno, L.; Aspillaga, H. y Biancant, F. Fibrosis Quística del Páncreas. Rev. Chil. Pediatr. 33:280, 1962 .

9 Bozzo, S.; Figueroa, M. y López, O. Determinación de electrolitos en el sudor. Pediatria 3:41, 1960.

9 Figueroa, C.; Larraín, F.; Danús, O. y Urbina, A. Estudio de la función pancreática en un caso de Fibrosis Quística del Páncreas. Pediatría 17:7, 1974.

10 Gibson, L.E. and Cooke, R.E. Test for concentration of electrolytes in sweat and cystic fibrosis of pancreas utilizing pilocarpine by ionthophoresis. Pediatrics 23: 545,1959 .

1 Domberger, G.R.; Comfort, M.W.; Wollaeger, $E$. E. and Power, M.H. Pancreatic function as measured by analysis of duodenal contents before and after stimulation with secretin. Gastroenterology 11:701, 1948.

12 Danús, O.; Hormazábal, J. Test de caroteno plasmático en el diagnóstico de la enfermedad celiaca. Correlación en la histología duodeno-yeyunal. Pediatría 17:13, 1974

13 Ceruti, E.; Contreras, J. y Neira, M. Sthaphylococcal pneumonia in childhood. Long-term follow-up including pulmonary function studies. Amer. J. Dis. Child. 122:386, 1971

14 Jackson, A.D. and Young, W.F. Cystic Fibrosis of the pancreas. Long-term observation of chronic diseases. Springer, Berlin, Gottingen and Heilderberg. pp. $178,1960$.
15 Huang, N.N. (ed.). Guide to Durg Theraphy in Patients with Cystic Fibrosis. Atlanta, Ga. Cystic Fibrosis Foundation, 1974.

I6 Tecklin, J.S. and Holsclaw, D. Evaluation of bronchial drainage in patients with cystic fibrosis. Phys. Ther. 55:1081, 1975 .

17 Schwachman, $H$. and Khaw, K-T. Cystic Fibrosis. IN Shirkey, H. Peddiatric Therapy. $5^{\circ}$ ed. C.V. Mosby St. Louis, 1975.

is Fanconi, G. und Wehlinger, E. und Knauer, C. Das Coelia-kiesyndrome bei angeborener zystischer pankreas fibroma tose und bronchiekstasien. Wien Med Wochenschr 86:753, 1936 .

19 Holsclaw, D.S.; Perlmutter, A.D.; Jackin, $H$ and Schwachman, H. Genital abnormalities in male patient with cystic fibrosis. J. Urol. 106:568, 1971.

20 Kaplan, E.; Schwachman, H.; Perimutter, A.D.; Rule, A.; Khaw, $K$. and Holsclaw, D. Reproductive failure in males with cystic fibrosis. New Engl. J. Med. 279:65, 1968.

21 Correa, M. y Lago, M.T. Tesis para optar al título de Kinesiólogo, 1977.

22 Harris, R.L. and Riley, H. Cystic Fibrosis in the American Indian. Pediatrics. 41:733, 1968.

23 Lloyd-Still, J.D.; Klaw, K.T y Schwachman, $H$. Severe Respiratory Disease in Infats with cystic fibrosis. Pediatrics. 53:678. 1974

24 Kopito, L.E.; Schwachmon, H.; Vawter, G.F. and Edlow, J. The pancreas in cystic fibrosis: Chemical composition and comparative morphology Pediatr. Res. 10:742, 1976.

25 Di Sant'Agnese, P.A. and Davis, P.B. Medical progress Research in cystic fibrosis. New Engl. J. Med. 295:481, 534, 597, 1976

2t Holsclaw, D.S. Common pulmonary complications of cystic fibrosis. Clin. Pediatr. 9:346, 1970.

27 Schwachman, H.; Redmond, A.; and Khaw, K-T. Studies in cystic fibrosis: Repon of 130 patients diagnosed under 3 months of age over a 20 year period. Pediatrics. 46:335, 1970.

28 Mellins, L.R.; Ingram, R. and Fishman, A. Obstructive disease of the airways in cystic Fibrosis. Pediatrcs. 41:560. 1968.

29 Cook, C.D. and Schwachman, H. Studies of Respiratory Physiology in Children: II. Lung volumes and mechanics of respiration in 64 patients with Cystic Fibrosis of the Pancreas. Pediatrics. 24: 181, 1959.

${ }^{30}$ Featherby, E.A.; Weng, T.R.; Crozier, D.N.; Duic, $A$.; Reilly, B.J. and Levison, $H$. Dynamic and static lung volume, blood-gas tensions, and diffusing capacity in patients with Cystic Fibrosis. Proc $5^{\text {th }}$ International Cystic Fibrosis Conference. Published by C.F. Research Trust. London, 1969

3 Godfrey, S. and Meams. M. Pulmonary Function and Responde to Exercise in Cystic Fibrosis. Arch. Dis. Child. 46:144, 1971.

32 Corey, M.; Levison, $H$. and Prozzer, D. Five to seven years course of pulmonary function in Cystic Fibrosis. Amer. Rev. Resp. Dis. 114:1085, 1976 
33 Donnison, A.; Schwachman, H. and Gross, R. A review of 164 children with meconium ileus. Pediatrics. 37:833, 1966.

34 Craig, J.; Haddad, H. and Schurachman, $H$. The pathological changes in the liver in Cystic Fibrosis of the Pancreas. Amer. J. Dis. Child. 93:337, 1957

35 Kopito, L.; Kosasky, $H$. and Schwachman, H. Water and electrolytes in cervical mucus from patients with Cystic Fibrosis. Fertil. Steril. 24:512, 1973.

36 Di Sant Agnese, P.A.; Darling, R.C.; Percre, G.A. and Shea, $E$. Abnormal electrolyte composition of sweat in cystic fibrosis of pancreas. Cinical significance and relationship to disease. Pediatrics. 12:549, 1953.
${ }^{37}$ Evaluation of testing for Cystic Fibrosis; report of the Committee for a study for evaluation of testing for Cystic Fibrosis. National Academy of Sciences, Washington. J. Pediatr. 88:711, 1976.

38 Sthephen, U.; Bush, E.W.; Kollberg, H.; and Hellsing, $K$. Cystic Fibrosis detection by Means of a Test-Strip. Pediatrics. 55:35, 1975.

39 Holsclaw, H.D.; Keith, H. and Palmer, J. Meconium Screening for Cystic Fibrosis. Pediatr. Ann. 7:15, 1978.

40 Schwachman, H.; Kozalski, M. and Khaw, T-G. Cystic Fibrosis: A new outlook. Medicine. 56:129, 1977. 